\title{
Performance tests of the MSMGRPCs using a free-streaming readout
}

\section{Petriş*! D.Bartoş, M. Petrovici, L. Rădulescu, V. Simion}

National Institute for Physics and Nuclear Engineering (IFIN-HH), Bucharest, Romania

E-mail: mpetrisanipne.ro

\section{J. Frünhauf, P.-A. Loizeau}

GSI Helmholtz Center for Heavy Ion Research GmbH (GSI), Darmstadt, Germany

\section{Deppner, N. Herrmann, C. Simon}

Physikalisches Institut der Universität Heidelberg, Heidelberg, Germany

\begin{abstract}
Result of the R\&D activity for the development of a Multi-Gap Resistive Plate Chamber with Multi-Strip readout architecture (MSMGRPC), for high counting rate and multiplicity environment, specific for high interaction rate experiments, are reported.

The free streaming readout concept considered for the CBM experiment at future FAIR facility in Darmstadt, Germany, requires MSMGRPCs with a very good matching of the signal transmission line impedance to the front-end electronics input impedance such that fake signals resulted from reflexions to be neglijible.

Two prototypes based on low resistivity glass $\left(\sim 10^{10} \Omega \mathrm{cm}\right)$ with the impedance of the signal transmission line matched to the input impedance of the front-end electronics were constructed. The granularity requirement for the inner zone of the CBM-TOF wall is fulfilled by a proper choice of the strip lenght. The prototypes were tested in-beam at CERN-SPS with reaction products produced by colliding $\mathrm{Pb}$ beam of momenta of $1 \cdot \mathrm{A} \mathrm{GeV}, 30 \cdot \mathrm{A} \mathrm{GeV}$ and $150 \cdot \mathrm{A} \mathrm{GeV}$ on a $\mathrm{Pb}$ target. The performance obtained using a free-streaming readout is reported.
\end{abstract}

ICHEP2018, International Conference on High Energy Physics

5-11 July 2018

Seoul, Korea

*Speaker.

${ }^{\dagger}$ A footnote may follow. 


\section{Introduction}

Compressed Baryonic Matter (CBM) experiment is a fixed target experiment, dedicated to the exploration of the phase diagram of strongly interacting matter in the region of high-net baryon densities and moderate temperatures. It is designed to run up to $10^{7}$ interactions per second. The physics program of the experiment is comprehensively detailed in [1]. The Time Of Flight (TOF) subsystem [2] is one of the core detectors of the CBM experimental setup. The TOF wall will be build based on multigap resistive plate chambers [3] with multistrip redout (MSMGRPC) [4]. In order to cope with the high counting rates anticipated to reach up to $30 \mathrm{kHz} / \mathrm{cm}^{2}$ at low polar angles (very close to the beam pipe), the counters of the innermost zone will be equipped with resistive electrodes made of low resistivity glass. In order to cope with such interaction rates, the data acquisition is based on self triggered electronics.

\section{Detector description}

Two new prototypes based on low resistivity glass $\left(\rho \sim 10^{10} \Omega \mathrm{cm}\right)$, with the impedance of the MSMGRPC signal transmission line matched to the input impedance of the front-end electronics were designed and built with the aim to fulfill the requirements of the inner zone of the CBMTOF wall. The transmission line impedance of a single readout channel of each of the developed prototypes was estimated using APLAC simulations [5, 6]. Both of them have the same active area of $96 \mathrm{~mm}$ width (along to the strips length) and $300 \mathrm{~mm}$ length. The first developed prototype has a classical single stack architecture (RPC2015SS) with 8 gas gaps of $140 \mu \mathrm{m}$. The readout and high voltage electrodes are designed with 28 strips with a pitch size of $10.2 \mathrm{~mm}$ and a strip width of $8.6 \mathrm{~mm}$ [7]. The second prototype has a double stack configuration (RPC2015DS) of $2 \times 5$ gas gaps of $140 \mu \mathrm{m}$. It was built based on an original developed method [5] which allows to tune the signal transmission line impedance to match any imput impedance of a given front-end electronics, independent on the granularity. The prototype was designed with the same pitch $(7.2 \mathrm{~mm})$ for both high voltage and readout electrodes, but with different values of the strip widths: $1.3 \mathrm{~mm}$ for the readout electrodes and of $5.6 \mathrm{~mm}$ for the high voltage ones [7]. Its active area is covered by 40 strips. The two MSMGRPCs are mounted in the same housing box, SSRPC2015 on top and DSRPC2015 on bottom.

\section{Experimental details}

The prototypes were tested in-beam at CERN-SPS with reaction products produced by $\mathrm{Pb}$ beam of momenta of $13 \cdot \mathrm{A} \mathrm{GeV}, 30 \cdot \mathrm{A} \mathrm{GeV}$ and $150 \cdot \mathrm{A} \mathrm{GeV}$ incident on a $\mathrm{Pb}$ target. The above mentioned prototypes were positioned in the experimental setup downstream relative to four MRPC modules developed for the outer zones of the TOF wall [8], details being shown in Fig. 1. A diamond detector, positioned in front of the target was used as beam reference. The signals delivered by the detectors were processed by PADIX [9] as front-end electronics. A free streaming data acquisition system (DAQ) was operated for the first time in an in-beam test. The DAQ system was equipped with GET4-TDCs [10] and used an AFCK data processing board [11] loaded with gDPB firmware [12]. A gas mixture of $85 \% \mathrm{C}_{2} \mathrm{H}_{2} \mathrm{~F}_{4}+5 \%$ iso- $\mathrm{C}_{4} \mathrm{H}_{10}+10 \% \mathrm{SF}_{6}$ was flushed through 


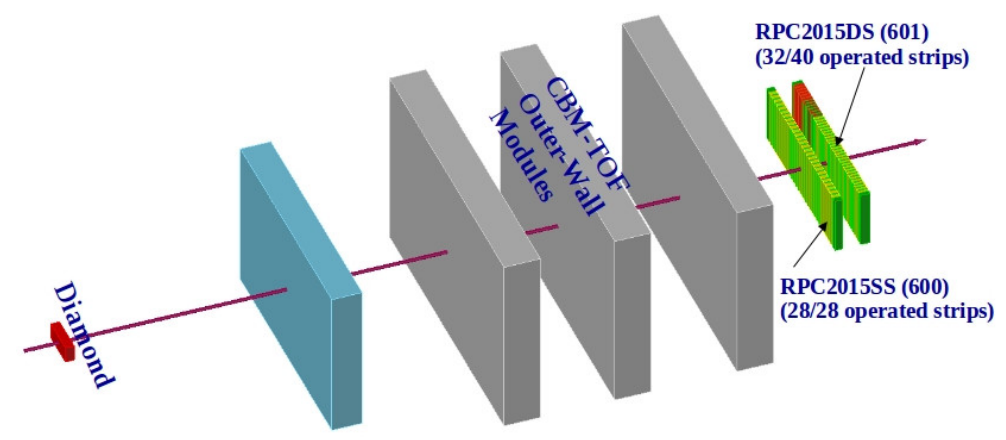

Figure 1: The position of the two MSMGRPCs in the experimental setup at CERN-SPS.

the counters at a $5 \mathrm{l} / \mathrm{h}$ flow, at atmospheric pressure. For the estimation of the time resolution and efficiency, RPC2015SS was considered as detector under test (DUT) and RPC2015DS as reference counter (REF).

\section{Results}

The system time resolution (the contribution of both DUT and REF counters) was estimated from the time difference between RPC2015SS and RPC2015DS, after corrections for the slewing effect, reaction product velocity spread and position in the reference counter. The obtained time spectrum fitted with Gauss function is presented in Fig.2, left side. A very good system time res-
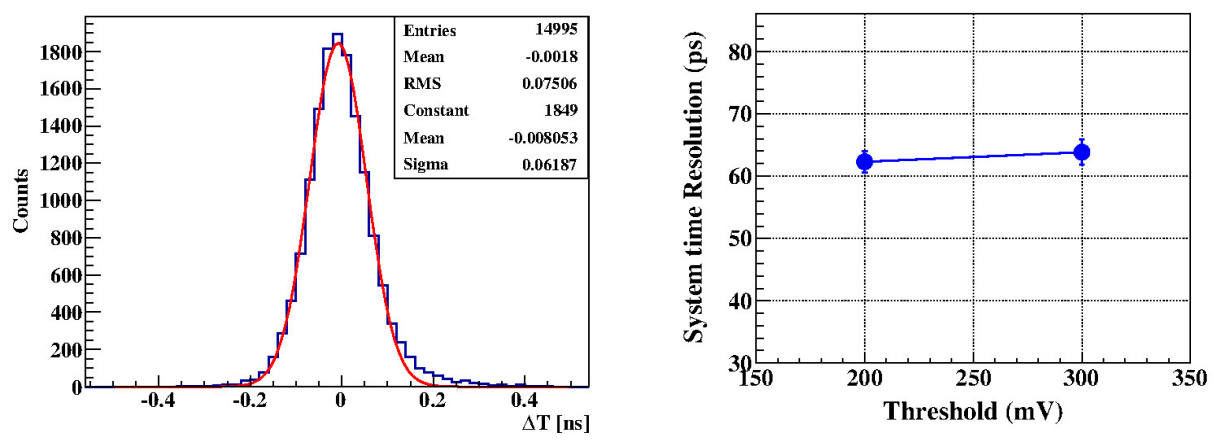

Figure 2: Left: Time difference between DUT and REF MSMGRPCs. Right: System time resolution as a function of PADIX threshold.

olution of $62 \pm 2 \mathrm{ps}$, including the contribution of the electronics, was obtained for operation of both RPC2015 at $157 \mathrm{kV} / \mathrm{cm}$, with the PADIX threshold set to $200 \mathrm{mV}$. A single counter resolution of 44 ps is obtained, if equal contributions of the two counters is considered. The system time resolution does not change significantly with PADIX threshold, as is shown in Fig. 2, right side. The obtained efficiency for the DUT was of 0.934 for $200 \mathrm{mV}$ electronics threshold. The reason of this lower value relative to the previous results $[13,14]$ obtained using a triggered DAQ system is under investigation. A cluster size of 2.0 strips was obtained for the RPC2015SS for the counter operation with $200 \mathrm{mV}$ electronics threshold (see Fig.3) and it decreases at 1.9 strips if the electronics threshold is increased at $300 \mathrm{mV}$. In Fig. 3 - left side, one can see that more than 3/4 of the events 

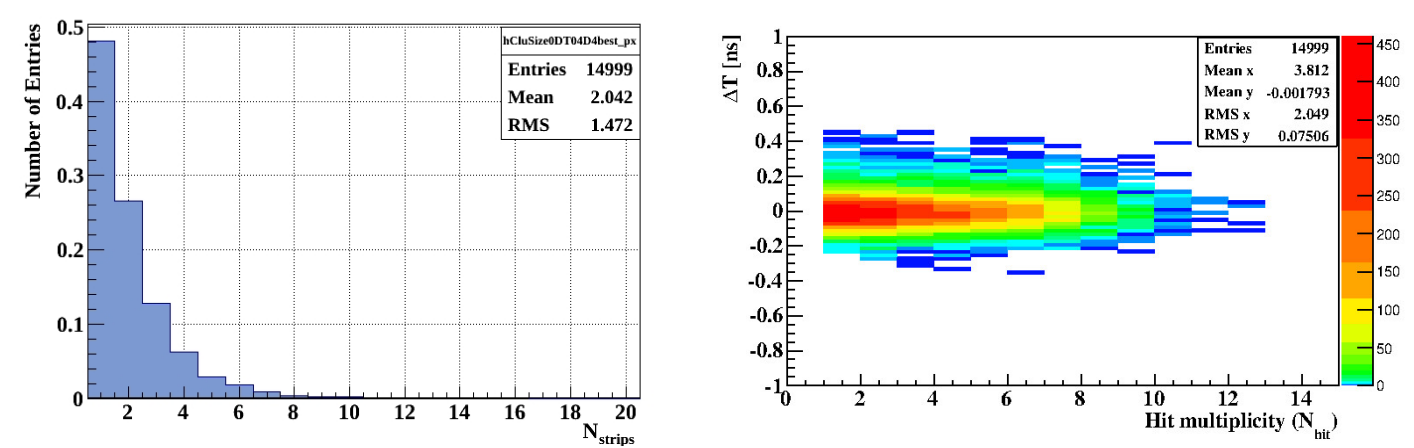

Figure 3: Left: Cluster size distribution for $200 \mathrm{mV}$ electronics threshold. Right: Correlation between time difference and number of hits per event.

have a cluster size of one or two strips. Fig. 3 - right side shows the correlation between the time difference and hit multiplicity. One can be seen that there were up to 8 hits incident simultaneously on the active area of the counter. For the average cluster size value of 2 strips, this gives a number of 16 strips with signal out of 28 total number of strips of DUT, a more than $50 \%$ occupancy of the counter.

\section{Conclusions}

The obtained results demonstrate the possibility to operate MSMGRPCs in a free-streaming readout mode with minimum fake signals produced by reflections, thus becoming a real candidate for high interaction rate experiments.

\section{References}

[1] CBM Collaboration, Eur. Phys. J. A, 53 (2017) 60. doi:10.1140/epja/i2017-12248-y.

[2] CBM - TOF Collaboration, CBM-TOF TDR, October 2014. https://repository.gsi.de/record/109024

[3] E. Cerron Zeballos et al., Nucl. Instrum. Meth. A, 374 (1996) 132.

[4] M. Petrovici et al., Nucl. Instrum. Meth. A, 487 (2002) 337.

[5] D. Bartoş et al., Romanian Journal of Physics 63 (2018) 901

[6] https://www.rfglobalnet.com/doc/aplac-800-student-version-0001

[7] V. Aprodu et al., CBM Progress Report 2015 (2016), p. 97 - 98

[8] I.Deppner, CBM Progress Report 2016 (2017), p.124

[9] M. Ciobanu et al., CBM Progress Report 2013, (2014), p.84

[10] https://wiki.gsi.de/pub/EE/GeT4/get4.pdf

[11] https://www.ohwr.org/projects/afck/wiki

[12] "CBM GitLab repository", https://cbmgsi.githost.io/p/loizeau/dpbcontrols

[13] M. Petrovici et al., JINST 7 P11003 (2012)

[14] M. Petriş et al., JINST 11 C09009 (2016) 